\title{
A Numerical Solution of Integral Equation of the First Kind Applied to Slit Correction in Small- Angle X-Ray Scattering
}

\author{
Jacob Mazur \\ Institute for Materials Research, National Bureau of Standards, Washington, D.C. 20234
}

(November 2, 1971)

\begin{abstract}
A novel approach to the problem of the slit correction in small-angle $\mathrm{x}$-ray scattering is presented, based on a matrix inversion method. The integral equation for the slit correction is written as a Volterra equation of the first kind. This equation is reduced to a system of simultaneous equations, expressed in matrix form. The order of the matrix is equal to the number of experimentally determined points. To obtain these equations, one has to expand the unknown functions in Taylor series around each point to be subsequently determined. There is, however, a difficulty inherent in this method due to the fact that most of the series expansions of the function to be determined lead to a strong numerical instability. However, a general method is developed, which enables us to find shifting operators leading to numerically stable systems of equations. The "unsmearing" of the experimental data is then performed by standard matrix inversion procedures.
\end{abstract}

Key words: Conditions for convergence of solutions; integral equation of first kind; shifting operators; slit correction; slit weighting function; small-angle $\mathrm{x}$-ray scattering; strong numerical instability.

\section{Introduction}

In a previous paper $[1]^{1}$ methods for solving the integral equation for slit correction in small angle x-ray scattering experiments were proposed. This equation relates the experimentally determined intensity of scattered ravs to the true scattering intensity as follows:

$$
\widetilde{I}(x)=2 \int_{x}^{\infty} \frac{y I(y) W\left(\sqrt{y^{2}-x^{2}}\right) d y}{\sqrt{y^{2}-x^{2}}} .
$$

In eq (1), $\widetilde{I}(x)$ is the experimentally determined intensity of the scattered light, $I(x)$ is the true scattering intensity, $x$ is proportional to the scattering angle [1], and $W(x)$ is the experimentally determined slit weighting function. The dependence of $W$ on the slit collimation system is widely discussed in references [2-5]. It may be given either in analytic form or only for discrete values of its argument.

$I(x)$ is to be calculated from eq (1), which is a Volterra integral equation of the first kind. In Reference [1] explicit and implicit solutions have been proposed. The explicit solution is based on transforming eq (1) into a Volterra equation of the second kind, which has for its inhomogeneous part a constant term, and whose kernel involves an integrated expression of the derivative of the experimentally determined slit weighting function $W$. This solution requires numerical differentiation of the experimental data, a process which may introduce serious errors. Differentiation is not required in the implicit solution method. Rather, the experimental data enter into the solution directly.

AMS Subject Classification: Primary 4510.

${ }^{1}$ Figures in brackets indicate the literature references at the end of this paper. 
This paper will deal only with the implicit solution method. In that case, eq (1) is first reduced to a system of simultaneous linear equations. These equations are then solved by inversion of the resulting triangular matrix. The solution leads to calculated approximate values of $I(x)$, given on a set of $N$ discrete points $x=0, x=\Delta x, \ldots ., x=(N-1) \Delta x$. These are the same points for which values of $\tilde{I}(x)$ must be given by experiment.

One of the difficulties in applying this procedure directly is that it can lead to numerical instability. However, one can find an interpolating polynomial for $I(x)$ such that a system of simultaneous equations is obtained which is numerically stable. In this paper will explore ways to find an accurate interpolating polynomial for the purpose of our computation. However, the problem of finding a suitable polynomial can be altogether avoided if another, indirect method is employed. That method consists in first obtaining a zeroth-order approximation to the solution, based on a single-step method and which is known to be always numerically stable. Subsequent improvements to the solution are then computed, using standard perturbation methods to obtain subsequent corrections to the zeroth-order approximation.

The first of these two approaches involves application of multi-step methods which are based on existence of polynomial which interpolates the function $I(x)$ at discrete points on a given interval of $x$. The second approach is based on a single-step method, which serves as a starting point for the perturbation method. Both approaches are included in a computer program that solves the problem of slit correction in small angle x-ray scattering. Four different choices for the weighting function $(W$ in eq $(1))$, have been used in that program:

1. Constant weighting function (infinite slit)

2. Gaussian

3. Trapezoid, and

4. Data weighting function.

The details of these computations and an analysis of the results will appear in an NBS Technical Note, to be published. In this Note, the Fortran program that performs the required calculations will be described in detail.

\section{Algebraic Formulation of the Problem}

The kernel of the integral eq (1) has a singularity at $y=x$. In order to deal with this singularity, we follow theprocedure employed in solving Abel's integral equation. Both sides of eq (1) are multiplied by $x d x / \sqrt{x^{2}-u^{2}}$ and integrated over $x$ from $u$ to infinity. The transformed equation, after exchanging the orders of integration is

$$
\int_{u}^{\infty} \widetilde{I}(x) G(u, x) d x=\int_{u}^{\infty} I(x) K(u, x) d x
$$

with

$$
K(u, x)=2 x \int_{0}^{\sqrt{x^{2}-u^{2}}} \frac{W(t) d t}{\sqrt{x^{2}-u^{2}-t^{2}}}
$$

and

$$
G(u, x)=x / \sqrt{x^{2}-u^{2}}
$$

The total number of experimental data for $\widetilde{I}(x)$ is $N$, which are given at equally spaced intervals $\Delta x$. The range of $x$ is determined in such a way that putting $\widetilde{I}(x)$ equal to zero for all $x \geqslant N \Delta x$ does not affect the solution. In the physical problem we are investigating, the assumption that $\widetilde{I}(x)=0$ for $x \geqslant N \Delta x$ implies that $I(x)=0$ for the same values of $x$. Therefore, both the upper limits of integrals in (2) are replaced by $N \Delta x$. Thus, the function $\widetilde{\widetilde{I}}(x)$ represents a discrete set of function values $\widetilde{\widetilde{I}}_{i}$, $i=1,2, \ldots ., N$. Both $\widetilde{I}(x)$ and $I(x)$ may therefore be viewed as $N$-dimensional vectors. The first components $F_{1}$ and $I_{1}$ of these vectors correspond to $u=0 ; i=N$ corresponds to $u=(N-1) \Delta x$. 
It should be remarked, that consistent with the above restrictions on the functions $\widetilde{I}(x)$ and $I(x)$, the method of solution presented in this paper is not necessarily restricted to the particular representations of $K(u, x)$ and $G(u, x)$, which in the problem being investigated are given by eqs (2a) and (2b). Hopefully, the method presented here is general and might be applied to other physical problems which can be simulated by eq (2).

The left-hand side of eq (2) is calculated from known quantities. The results are represented by the vector $\mathbf{h}$ with components

$$
h_{i}=\int_{(i-1) \Delta x}^{N \Delta x} \tilde{\widetilde{I}}(x) G(u, x) d x
$$

In our particular problem, $G(u, x)$ is singular at $x=u$ and is given by eq $(2 \mathrm{~b})$. For this reason, a direct numerical evaluation of the integral (3) will not be practical, since we will have to subdivide the integrand very closely at its lower end in order to achieve sufficient accuracy. To evaluate (3) we replace the integral by summation as follows:

$$
\int_{u}^{\infty} \frac{x \widetilde{I}(x) d x}{\sqrt{x^{2}-u^{2}}} \cong \sum_{j=i}^{N} \int_{(j-1) \Delta x}^{j \Delta x} \frac{x I(x) d x}{\sqrt{x^{2}-(i-1)^{2} \Delta x^{2}}} .
$$

Next, $\widetilde{I}(x)$ is expanded in a Taylor series around $x=j \Delta x$ :

$$
h_{i}=\sum_{j=i}^{N} \int_{(j-1) \Delta x}^{j \Delta x}\left[I(j \Delta x)+(x-j \Delta x) A_{1}+\frac{1}{2}(x-j \Delta x)^{2} A_{2}+\ldots\right] \frac{x d x}{\sqrt{x^{2}-(i-1)^{2} \Delta x^{2}}}
$$

$A_{1}, A_{2}$, etc., are the first, second, etc. derivatives of $\widetilde{I}(x)$ evaluated at $x=j \Delta x$. These derivatives can be approximated by one of several difference relations. We may use the following finite difference expression:

$$
A_{i}=\left(\tilde{\widetilde{I}}_{j+1}-\tilde{\widetilde{I}}_{j}\right) / \Delta x, \quad A_{2}=\left(\tilde{\widetilde{I}}_{j+2}-2 \tilde{\widetilde{I}}_{j-1}+\tilde{\widetilde{I}}_{j}\right) /(\Delta x)^{2} .
$$

Only two derivatives are used in the Taylor series in (5); $\widetilde{\widetilde{I}}_{N+1}$ and $\widetilde{\widetilde{I}}_{N+2}$ are set equal to zero. After performing the indicated integrations and rearranging eq (5), the result is written in the form

$$
h_{i}=\sum_{j=i}^{N} \gamma_{i, j} \widetilde{I}_{j} ; \quad \widetilde{I}_{j}=0 \text { for } j>N .
$$

or $\mathbf{h}=\mathbf{A} \tilde{\mathbf{I}} . \mathbf{A}$ is an upper triangular matrix with entries the coefficients $\gamma_{i, j}$. The matrix elements $\gamma_{i, j}$, have different representations depending on the following three cases: (1) $j-i=0,(2) j-i$ even, and (3) $j-i$ odd. In addition, in order to close the system, one has to consider, separately, the cases $j=N$ and $j=N-1$. This is because the last integral, if taken from $x=(N-1) \Delta x$, cannot include second-order differences if forward extrapolation is employed. Also for the case $i=1(u=0)$ we have to avoid the expression $(i-1) \log (i-1)$. Details will be given in an NBS Technical Note describing the computer program.

We now expand the r.h.s. of the integral equation (2) in terms of the discrete values $I_{i}$, as

$$
\int_{(i-1) \Delta x}^{N} I(x) K(u, x) d x=\sum_{j=i}^{N} b_{i, j} I_{j} ; \quad I_{j}=0 \quad \text { for } \quad j>N .
$$

to obtain a set of simultaneous equations

$$
\sum_{j=1}^{N} b_{i, j} I_{j}=h_{i}
$$


$b_{i},{ }_{j}(i=1,2, \ldots ., N ; j=i, i+1, \ldots ., N)$ are coefficients which we have not yet determined. These coefficients depend on the functional form of the kernel $K(u, x)$, and, therefore, on the assumed form for the weighting function $W$ of eq (1). In addition, they also depend on the coefficients of the interpolating polynomial which approximates the function $I(x)$.

The solution of eq (8) can be expressed in matrix form:

$$
\mathbf{I}=\mathbf{B}^{-1} \mathbf{h} \text {. }
$$

I is the $N$-dimensional solution vector. $\mathbf{h}$ is an $N$-dimensional vector whose elements were already computed from the experimental data and the indicated numerical integrations. The inversion of an upper triangular matrix $\mathbf{B}$ can be rapidly performed by a back substitution [5], starting with the last equation. With this method, the elements $c_{i, j}$ of the inverse matrix $\mathbf{B}^{-1}$ are obtained by first calculating the elements $d_{i, j}$ from the following recurrence equations:

$$
\begin{aligned}
& d_{i, i}=1, \\
& d_{i, j}=\sum_{k=i}^{k=j-1}-\left(\frac{b_{k, j}}{b_{i, k}}\right) d_{i, k}
\end{aligned}
$$

and then

$$
c_{i, j}=d_{i, j} / b_{i, j} .
$$

For certain forms of $K(u, x)$, the kernel of the r.h.s. of eq (2), there is a repetitious pattern in the representations of the elements $d_{i, j}$. In this case, the number of different elements in the matrix $\mathbf{B}$ is less than $(1 / 2) N(N+1)$. In particular, if $K(u, x)$ is independent of $u$ (e.g., when $W$ of eq (2a) is constant and therefore, $K(u, x)=2 x)$ the elements of the matrix $\mathbf{B}$ (after multiplication by the diagonal matrix

$$
\left\{\frac{1}{i} \delta_{i j}\right\}
$$

depend only on $|j-i|$. This has an important practical aspect: computer computations with large matrices require storage capacity which is often not available. It is then necessary to employ vectors $\mathbf{b}_{j}$ (formed separately for each row of the matrix $\mathbf{B}$ ), or, to compute $b_{i, j}$ each time it appears in computations, separately. The problem is, that these computations will be repeated many times. However, for this case, only the first row of the matrix, in form of a vector $\mathbf{b}_{N}$ needs to be computed, and the problem of storing large matrices is avoided.

Any algorithm in which the calculated value of the increment $I_{i}-I_{i+1}$ depends only on $I_{i+1}$ and on the step $\Delta x$, is called a one-step method. In other words, an expression for $I_{i}$ may be written in a closed form involving explicitly only $I_{i+1}$ Of course, $I_{i+1}$ depends, in its turn, on $I_{i+2}$, etc. In a multi-step method of multiplicity $l$, the increment $I_{i}-I_{i+1}$ depends also on $I_{i+2}, \ldots, I_{i+l}$. It therefore follows, that whenever one approximates $I(x)$ in eq (1) by an interpolating polynomial of order higher than one, the method of calculating $I_{i}$ in terms of already computed $I_{i+1}, I_{i+2}$, etc., will be defined as a multi-step method of multiplicity which is equal to the order of the interpolating polynomial. In a multi-step method, a special starting procedure is required to find the "initial" or "starting" values of $I_{N}, I_{N-1}, \ldots, I_{N-l}$. We calculate these starting values by trapezoid rule as follows:

$$
\begin{gathered}
h_{N-1}=\frac{1}{2} \int_{(N-2) \Delta x}^{(N-1) \Delta x}\left(I_{N}+I_{N-1}\right) K[(N-2) \Delta x, x] d x+\frac{1}{2} I_{N} \int_{(N-1) \Delta x}^{N \Delta x} K[(N-2) \Delta x, x] d x \\
2 h_{N-l}=I_{N-l} \int_{(N-l-1) \Delta x}^{(N-l) \Delta x} K[(N-l-1) \Delta x, x] d x+I_{N-l+1} \int_{(N-l-1) \Delta x}^{(N-l+1) \Delta x} K[(N-l+1) \Delta x, x] d x \\
\ldots+I_{N} \int_{(N-2) \Delta x}^{N \Delta x} K[(N-l-1) \Delta x, x] d x
\end{gathered}
$$


We have $l$ equations and $l+1$ unknowns. However, a physical assumption was made that setting $h=0$ for $i>N$, implies that $I_{i}=0$ for $i>N$. For this reason,

$$
I_{N}=2 h_{N} / \int_{(N-1) \Delta x}^{N \Delta x} K[(N-1) \Delta x, x] d x
$$

Let us divide the integral $h_{i}$

$$
h_{i}=\int_{(i-1) \Delta x}^{(N-1) \Delta x} I(x) K[(i-1) \Delta x, x] d x
$$

into smaller integrals, each with a range of integration equal to $l \Delta x$ (except for the last integral). For this purpose, let us define

$$
\kappa_{i, j}=\int_{(j-1) \Delta x}^{N \Delta x} I(x) K[(i-1) \Delta x, x] d x \quad(j=i, i+1, \ldots, N-1)
$$

so that $\kappa_{i, i}=h_{i}$.

$h_{i}$ is the sum of differences of the $\kappa_{i, j}$ as follows:

$$
h_{i}=\left(\kappa_{i, i}-\kappa_{i, i+l}\right)+\left(\kappa_{i, i+l}-\kappa_{i, i+2 l}\right)+\ldots . .
$$

(In this expression, the number of bracketed terms is equal to the nearest integer not exceeding $(N-i) / l$. In addition, a term is added to close the system so that $h_{i}=\kappa_{i}, i$.) Next, in each of the difference equations

$$
\kappa_{i, j}-\kappa_{i, j+l}=\int_{(j-1) \Delta x}^{(j+l-1) \Delta x} I(x) K[(i-1) \Delta x, x] d x
$$

we replace $I(x)$ by an interpolating polynomial having the values $I_{k}=I\left(x_{k}\right)$ on a set of points $x_{k}$. These points might be taken as $x_{k}=(j-1) \Delta x, \ldots .,(j-l-1) \Delta x$. In forward interpolation, the interpolating points are the $p$ points $j-1+m, j+m, . . ., j+m+p-1$, with $p \geqslant l$. $m$ denotes the point from which the differences are computed. We will always have $m<l$, so that the point of reference lies within the range of the integral (12). The polynomial $Q(x)$ which approximates and replaces $I(x)$ in eq (12), in terms of forward differences [6], is

$$
Q(x)=\sum_{q=0}^{p}\left(\begin{array}{c}
(x / \Delta x)-j-m+1 \\
q
\end{array}\right) \Delta^{q} I_{j+m} .
$$

In eq (13), $\Delta$ is a forward difference operator. Thus, for $q=1, \Delta I_{j+m+1}=I_{+m+1}-I_{j+m}$, etc. $\Delta^{\prime} I_{j+m}=\Delta I_{j+m}$.

Substituting eq (13) in eq (12), integrating and rearranging, the result is formally written as

$$
\kappa_{i, j}-\kappa_{i, j+l}=\alpha_{i, j} I_{j+m}+\alpha_{i, j+1} I_{j+m+1}+\ldots+\alpha_{i, j+p} I_{j+m+p}
$$

$\alpha_{i, j+k}(k=0,1, \ldots, p)$ are the coefficients resulting from the above steps taken in order to approximate eq (12) by eq (14).

In the formal solution, written in matrix form as $\mathbf{I}=\mathbf{B}^{-1} \mathbf{h}$, the elements of the matrix $\mathbf{B}$ can be expressed in terms of $\alpha_{i, j}$ of expansion (14).

Before proceeding, let us stop and see what will happen to our computations with $I(x)$ of eq (12) being replaced by polynomial $Q(x)$, for the specific case of $m=0$ and $l=p=2$. In this case, 
all three interpolated values of $I_{i}$ are within the range of the integral of eq (12). Let us assume that $K(u, x) \propto x$. This is the case when the weighting function $W$ is constant ( $W=$ constant is descriptive of an infinitely-wide slit in small angle $\mathrm{x}$-ray scattering). For $i \gg 1$, the elements of a typical row of the $\mathbf{B}$ matrix are found to be

$$
b_{i, i}=\frac{i}{3} ; b_{i, i+1}=\frac{4}{3}(i+1) ; b_{i, i+2}=\frac{2}{3}(i+2) .
$$

The coefficients $\alpha_{i, j}$ of eq (13) are:

$$
\alpha_{i, i}=\frac{i}{3} ; \alpha_{i, i+1}=\frac{4}{3}(i+1) ; \alpha=\frac{1}{3}(i+2) .
$$

The absolute values of the elements of the inverse of the $\mathbf{B}$ matrix can be shown to grow exponentially as one moves away from the diagonal elements. Consequently, the numerical calculations are affected by strong numerical instability.

\section{Stability Conditions for Integral Equations of First Kind}

\subsection{General Theory}

Consider first the difference eq (14) written for $\kappa_{i, i}-\kappa_{i, i+l}$ and for $p=l$. The associated homogeneous equation, obtained by setting

$\kappa_{i, i}-\kappa_{i, i+l}=0$, is

$$
\alpha_{i, i} I_{i+m}+\alpha_{i, i+1} I_{i+m+1}+\ldots .+\alpha_{i, l} I_{i+m+l}=0 .
$$

Solution of (15) can be found for every choice of starting values $I_{N}, I_{N-1}, \ldots, I_{N-l+1}$. (These are computed using the trapezoid rule and the one-step method.) In particular, if these starting values are all equal to zero, then there corresponds a trivial solution $I_{i}=0$ for every $i$.

Consider first a simplified homogeneous equation for the starting values of $\mathbf{I}$, with constant coefficients, replacing eq (15),

$$
\alpha_{0} I_{N-l}+\alpha_{1} I_{N-l+1}+\ldots+\alpha_{l} I_{N}=0 .
$$

We determine the conditions for convergence of solution of eq (16) for $N \rightarrow \infty$, while retaining finite value for $N \Delta x$. That is, the intervals between successive points becomes smaller and smaller as $N$ increases, and, as $N \rightarrow \infty, \Delta x \rightarrow 0$. If the multi-step method for solving eq (16) converges, then it will converge also for the special value of $I_{N}=0$.

Therefore,

$$
\lim _{\Delta x \rightarrow 0} I_{\mu}=0, \quad \mu=N, \quad N-1, \ldots, \quad N-l
$$

is a condition for convergence of multi-step method for solving eq (16). Accordingly, if condition (17) is not satisfied, the solution of the general homogeneous eq (15) will diverge in the limit of $\Delta x \rightarrow 0$. This divergence implies the divergence of the solution of the inhomogeneous eq (14). The procedure to determine the conditions for convergence of solution of eq (15) in the limit of $\Delta x \rightarrow \infty 0$ (or $N \rightarrow \infty$ ), is identical with the one employed in connection with numerical solution of difference-differential equations [7], and proceeds as follows: The homogeneous eq (15), with constant coefficients, is expressed as:

$$
\alpha_{i} I_{i+m}+\alpha_{i+1} I_{i+m+1}+\ldots .+\alpha_{i+l} I_{i+m+l}=0 .
$$


In eq (18), we substitute

$$
I_{i+m+l}=\eta^{-(i+m+l)} .
$$

Equation (18) becomes after division by common factor $\eta^{-(i+m+l)}$ :

$$
\alpha_{i} \eta^{l}+\alpha_{i+1} \eta^{l-1}+\ldots+\alpha_{i+l}=0 .
$$

Therefore, $\eta$ must be a root of the characteristic polynomial $\varphi(\eta)$ :

$$
\emptyset(\eta)=\alpha_{i} \eta^{l}+\alpha_{i+1} \eta^{l-1}+\ldots+\alpha_{i+l}=0 .
$$

We require that the multi-step method be convergent. The necessary condition for the convergence of the general multi-step method for our integral equation of the first kind is the same as the condition for the convergence of difference equations of first order [8], and is stated by the following:

THEOREM: A necessary condition for the convergence of the multi-step solution (14) for integral equation ( 1 ) is that the moduli of all roots of the associated polynomial ${ }^{\circ}(\eta)$ do not exceed unity, and that the root of modulus 1 is simple.

The condition imposed on $\emptyset(\eta)$ is also the condition of stability [9]. Violation of this condition leads to "strong" numerical instability. This instability manifests itself by the error growth at a fixed point $x=(i-1) \Delta x$ as the spacing between the points decreases. Strong instability is therefore characterized as a pointwise phenomenon, as it involves error growth at fixed points as the interval between them decreases. In contradistinction to this, a "weak" instability, to be discussed subsequently in connection with even-odd oscillations in computed values of $I_{i}$, is associated with the truncation error, and exists even when the method employed is convergent. Since the weak instability is associated with the total range of $x$, while keeping the interval between successive points constant, this instability is also known as a stepwise phenomenon [7, 10].

Let us demonstrate the concept of strong instability by a concrete example. Assume that $I=2$ and $m=0$. Also, let $K(u, x) \propto x$, so that $\kappa_{i, j}-\kappa_{i, j+2}=h_{i}-h_{i+2}$. We substitute eq (13) into eq (12) and integrate. Since the r.h.s. of eq (12) is a function of $j$ only, $\alpha_{i, j}$, the elements of the matrix $\mathbf{B}$, are shown to be equal to $j \bar{\alpha}_{|j-i|}$ for $j \geqslant i$ and to zero for $j<i . \bar{\alpha}_{|j-i|}$ is a function of $j-i$ only. If, in addition, $p=l$, then, for $i \gg 1$,

$$
h_{i}-h_{i+2}=\alpha_{0} I_{i}+\alpha_{1} I_{i+1}+\alpha_{2} I_{i+2}
$$

where

$$
\alpha_{0}=\alpha_{i, i} \cong i \bar{\alpha}_{0}, \alpha_{1}=\alpha_{i, i+1} \cong i \bar{\alpha}_{1}
$$

and

$$
\alpha_{22}=\alpha_{i, i+2} \cong i \bar{\alpha}_{2} .
$$

For a particular case when $\alpha_{0}=\alpha_{2}$ (as in the example discussed at the end of chap. 2), the elements $b^{*}{ }_{i, j}$ of the inverse matrix $\mathbf{B}^{-1}$ can be expressed directly in terms of the roots of the characteristic polynomial $\varphi(\eta)$, eq (19). One obtains

$$
\underset{(j>i)}{i b_{i, j}^{*}}=\left[\frac{1}{2 \alpha_{0}}\left(-\alpha_{1}+\sqrt{\alpha_{1}^{2}-4 \alpha_{1} \alpha_{0}}\right)\right]^{k}+\left[\frac{1}{2 \alpha_{0}}\left(-\alpha_{1}-\sqrt{\alpha_{1}^{2}-4 \alpha_{1} \alpha_{0}}\right)\right]^{k}
$$

and $b_{i, i}^{*}=1 / i$. 
In eq (22), $k=j-i$. When $\alpha_{2} \neq \alpha_{0}$, the relationships between $b^{*}{ }_{i, j}$ and the roots of eq (19) are more involved. However, for sufficiently large $k$, the leading term in the expression for $b^{*}{ }_{i, k+i}$ can be shown to be equal to

$$
\left|\frac{-\alpha_{1}}{2 \alpha_{0}}\right|^{k}
$$

For the case of $K(u, x) \propto x$, the following equation is derived

$$
I_{i}-I_{i+2}=\sum_{j=i}^{N} b^{*}{ }_{i, j}\left[h_{j}-(i /(i+2)) h_{j+2}\right] .
$$

When $k$ becomes infinitely large $I_{i}$ will remain finite only if $\lim _{(j-i) \rightarrow \infty} b^{*_{i, j}}$ converges. This happens only when both expressions in parentheses in eq (22) do not exceed 1 in their absolute values. This implies that the moduli of the roots of the equation

$$
\emptyset(\eta)=\eta^{2}+\alpha_{1} \eta+\alpha_{2}=0
$$

do not exceed unity. This simple example demonstrates the intimate connection between the root condition and the stability of the multi-step method.

In the general theory of stability of the difference differential equations, the necessary and sufficient conditions for the convergence of the multi-step method require, in addition to the root condition, also the satisfaction of the consistency condition [9]. This condition requires that, in order that an expansion in discrete variables (e.g., our expansion eq (14)) be a good approximation to the original equation which it simulates, certain restrictions must be imposed on the coefficients in such expansion. In our problem the consistency condition requires that the sum of the coefficients in eq (18) be equal to $l$ :

$$
\sum_{r=1}^{r=i+l} \alpha_{r}=l
$$

Since this condition is always satisfied, the root condition becomes both the necessary and the sufficient condition for the convergence of the multi-step method. We also see why the method for the determination of the starting values of $I_{i}$, based on a one-step method, is always stable and convergent: The polynomial $\emptyset(\eta)$ is then of the first order with its root equal to 1 .

\subsection{Determination of Stability Conditions}

Our purpose is to obtain an approximation to the integral

$$
\int_{(i-1) \Delta x}^{(i+l-1) \Delta x} I(x) K[(i-1) \Delta x, x] d x=\kappa_{i, i}-\kappa_{i, i+l}
$$

in terms of finite differences, which satisfies the stability conditions. These differences can be referred to the point $x=(i-1) \Delta x$, to the point $x=i \Delta x$, and so on. Let the point of reference be located at $x=(i+m-1) \Delta x, m=0, \ldots, l$. We define a shift (displacement) operator $\mathbf{E}$ by

$$
\mathbf{E} I_{j}=I_{j-1} .
$$

Applying the shifting operator to the point of reference, one obtains

$$
I(x)=\mathbf{E}^{-t}\left(I_{i+m}\right) .
$$

$t$ is a new variable, defined by $t=x / \Delta x-(i+m-1)$, and it measures the distance from the point of reference. Therefore, 


$$
\kappa_{i, i}-\kappa_{i, i+l}=\Delta_{i} \int_{-m}^{(l-m)} K\left(i^{*}, i^{*}+t^{*}+m^{*}\right) \mathbf{E}^{-t}\left[I_{i+m-1}\right] d t
$$

In eq (27) and in subsequent equations, $i^{*}=(i-1) \Delta x, t^{*}=t \Delta x$, and $m^{*}=m \Delta x$ and $K\left(i^{*}, i^{*}+\right.$ $\left.t^{*}+m^{*}\right)=K[(i-1) \Delta x,(i+t+m-1) \Delta x]$. The operator $\mathbf{E}$ can be expressed in terms of one of the three difference operators, $\boldsymbol{\Delta}, \boldsymbol{\nabla}$, and $\boldsymbol{\delta}$ which are defined by

$\Delta I_{j}=I_{j+1}-I_{j}$ (forward difference operator),

$\Delta I_{j}=I_{j}-I_{j-1}$ (backward difference operator),

$\boldsymbol{\delta} I_{j}=I_{j+1 / 2}-I_{j-1 / 2}$ (central difference operator).

These operators are related to $\mathbf{E}$ through

$$
\boldsymbol{\Delta}=1-\mathbf{E}, \boldsymbol{\Delta}=\mathbf{E}^{-1}-1, \boldsymbol{\delta}=\mathbf{E}^{1 / 2}-\mathbf{E}^{-1 / 2}
$$

For the backward difference operator $\Delta$ we have the following eq:

$$
\kappa_{i, i}-\kappa_{i, i+l}=\Delta x \int_{-m}^{l-m} K\left(i^{*}, i^{*}+t^{*}+m^{*}\right)\left[(1-\boldsymbol{\Delta})^{-t} I_{i+m-1}\right] d t .
$$

For forward difference operator $\boldsymbol{\Delta}$ we have

$$
\kappa_{i, i}-\kappa_{i, i+l}=\boldsymbol{\Delta} x \int_{-m}^{l-m} K\left(i^{*}, i^{*}+t^{*}+m^{*}\right)\left[(1+\Delta) t I_{i+m-1}\right] d t .
$$

In order to derive an equation for $\kappa_{i, i}-\kappa_{i, i+l}$ for central differences in terms of difference operator $\boldsymbol{\delta}$, we have for express $\mathbf{E}$ in terms of $\boldsymbol{d}$. Let $\mathbf{E}=e^{\mathbf{D}}$, and, therefore, $\boldsymbol{\delta}=2 \sinh (\mathbf{D} / 2)$. Thus,

$$
\begin{gathered}
\mathbf{D}=2 \sinh ^{-1}(\boldsymbol{\delta} / 2)=\boldsymbol{\delta}-\frac{\boldsymbol{\delta}^{3}}{2^{2} 3 !}+\frac{3^{2} \boldsymbol{\delta}^{5}}{2^{4} 5 !}+\ldots \\
\mathbf{E}^{-t}=1-t\left(\boldsymbol{\delta}-\frac{\boldsymbol{\delta}^{3}}{3 ! 2^{2}}+\ldots\right)+\frac{t^{2}}{2}\left(\boldsymbol{\delta}-\frac{\boldsymbol{\delta}^{3}}{3 ! 2^{2}}+\ldots\right)^{2} .
\end{gathered}
$$

Retaining terms involving no higher operators than $\boldsymbol{\delta}^{2}$, we have $\mathbf{E}^{-t}=1-\boldsymbol{\delta} t+t^{2} \boldsymbol{\delta}^{2} / 2$.

Therefore,

$$
\kappa_{i, i}-\kappa_{i, i+2}=\Delta x \int_{-m}^{I-m} K\left(\dot{i}^{*}, i^{*}+t^{*}+m^{*}\right)\left[\left(1-\boldsymbol{\delta} t+\frac{t^{2} \boldsymbol{\delta}^{2}}{2}\right) I_{i+m-1}\right] d t
$$

Let us now investigate a few cases with simplified kernels, and find out which ones lead to numerically stable solutions. For the kernel $K\left(i^{*}, i^{*}+t^{*}+m^{*}\right)$ we again adopt the form which is descriptive of the constant weighting function in small-angle $\mathrm{x}$-ray scattering, that is, $K\left(i^{*}, i^{*}+t^{*}+m^{*}\right)$ $=(i+t+m-1) \Delta x$. For the first case let us take the form is which the approximating polynomial agrees with $I(x)$ at $x=(i-1) \Delta x, \ldots,(i+l-1) \Delta x$. We take $l=2$, and $m=2$. In order that all points will fall within the range of the integral, we must use the $\nabla$ (backward) operator. Consider $i \gg 1$. The root-determining equation is $1 / 3\left(x^{2}+4 x+1\right)=0$. (The coefficient of $x^{2}$ is the coefficient of $I_{i}$, the coefficient of $x$ is the coefficient of $I_{i+1}$, and the last term is the coefficient of $I_{i+2}$.) We detect immediately that this form leads to a divergent solution. (Root condition is not satisfied.) 
Let us now consider the case with central differences centered about the mid-point of the integrand. Let $l=2$, and $m=1$. From eq (31), the following root-determining equation is derived:

$$
1 / 3 x^{2}+4 / 3 x+1 / 3=0
$$

and the method is unstable. It is interesting to notice that this particular case, which is the most obvious one since it is symmetric and does not involve odd powers of $t$ (in the integrand of eq (31), leads to an equation which is equivalent to the extended Simpson rule for numerical integration. The same result would be obtained if we evaluated the integral as

$$
\int_{i \Delta x}^{N \Delta x} f(i \Delta x, x) d x=1 / 3\left(f_{i, i}+4 f_{i, i+1}+2 f_{i, i+2}+\ldots\right) \Delta x
$$

with $f(i \Delta x, x)=K(i \Delta x, x) I(x)$, etc.

At this point, we are led to the conjecture that the most straightforward cases, namely the ones that involve points located within the range of the integral, lead to unstable solutions. If the above restriction is removed, stability conditions may be satisfied. For example, in the case with $m=2$, (the finite differences refer to the point $x=(i+1) \Delta x$ ) we have, for $l=2$ and the $\Delta$ operator, two fixed points which we located outside the range of the integral. The root condition can be shown to be satisfied.

The method that we find to be the best for our purposes is the one in which the $\Delta$ operator is used and the absolute values of the coefficients of $\boldsymbol{\Delta}^{r}, r=1,2, \ldots, p$, are made as small as possible. This can be achieved by selecting the point of reference to be located in the middle of the integrand, i.e., by taking $m=l / 2$. In this case, one is justified in retaining only the first few terms in the expansion of (27). We have, for $l=2$,

$$
\kappa_{i, i}-\kappa_{i, i+2}=\Delta x \int_{-1}^{1} K\left(i^{*}, i^{*}+t^{*}\right)\left[1-t \Delta+\frac{t}{2}(t-1) \Delta^{2}-. .\right] I_{i} d t
$$

For $K\left(i^{*}, i^{*}+t^{*}\right)=(i+t) \Delta x$, and $i \gg 1$, the coefficients of $I_{i+j}(j=0,1$ or 2$)$ in the expansion (34), after integration, are identical to the ones employed in Nÿstrom's method [11]. Since the root determining equation is

$$
7 / 3 x^{2}-2 / 3 x+1 / 3=0
$$

the root conditions are satisfied. We found that, of all the multi-step methods, the above method is the most accurate for our purposes. Part of the computer program for the slit correction in smallangle $\mathrm{x}$-ray scattering is based on this algorithm.

In conclusion, to insure stability, the differences in the expansion of the shifting operator should be referred to the point $\left(I_{i}\right)$ to be calculated and forward differences should be used. We also conjecture that the central difference method, which is the most accurate one in solving ordinary difference differential equation, is always numerically unstable.

The following qualitative considerations indicate that the stability conditions are only slightly altered by the actual form of $K[(i-i) \Delta x, x]$ and that a non-constant weighting function relaxes the condition for the attainment of the stability conditions. Consider $K(u, x)=x F\left(\sqrt{x^{2}-u^{2}}\right)$, (which is the form used in actual computations of slit corrections). $F$ is related to the weighting function $W$ by

$$
F\left(\sqrt{x^{2}-u^{2}}\right)=\int_{0}^{\sqrt{x^{2}-u^{2}}} \frac{W(y) d y}{\sqrt{x^{2}-u^{2}-y^{2}}}
$$


Now, let us form a matrix of $b_{i, j}$, where $b_{i, j}$ are the coefficients in the approximation of

$$
\int_{u}^{N \Delta x} x I(x) F\left(\sqrt{x^{2}-u^{2}}\right) d x \text { by an expansion } \sum_{j \geq i}^{N} b_{i, j} I_{j} .
$$

Consider, for example, a trapezoid form of $W(x)$. It can be shown that in each row of the matrix $\mathbf{B}=\left(b_{i, j}\right), b_{i, j}$ will decrease as $j$ increases, as compared with the case of constant $W$. This will result in a decrease of the ratio of the first to the last coefficients in the root-determining equation, and, therefore, in a decrease in the value of the modulus of the largest root of the polynomial, eq (20).

Thus, the stability conditions severely restrict us in selecting the best procedure for numerical computations of a system of simultaneous equations which replace the original integral equation of the first kind. This stability problem can be almost completely eliminated if the original integral equation of the first kind is transformed into an equation of the second kind, by differentiation. Then eq (21) becomes

$$
-K(u, u) I(u)+\int_{u}^{N \Delta x} I(x) \frac{d}{d u} K(u, x) d x=\frac{d h u}{d u} .
$$

If $K(u, u)=0$, the differentiation is repeated, until one obtained

$$
\left.\frac{d^{n}}{d u^{n}} K(u, x)\right|_{x=0} ^{\neq 0}
$$

When eq (37) is replaced by a set of simultaneous linear equations, the presence of the first term in the 1.h.s. of eq (37) drastically relaxes the restrictions imposed by stability consideration: The reason is that the presence of this term increases the values of the diagonal terms in the $\mathbf{B}$ matrix. This, in turn, tends to decrease the modulus of the largest root of eq (19). For this reason, it is preferable to transform eq (2) into eq (37) whenever $\tilde{\widetilde{I}}(u)$ and $K(u, x)$ are given as analytical functions. However, in our problem these functions are given as sets of points based on experimentally determined data. Their differentiations would introduce a further uncertainty in the computed results. The method of solution we developed is to be preferred only if the numerical differentiations of experimental data are to be avoided.

\section{Perturbation Methods}

One would expect that, of all difference approximations to derivatives, the ones based on central differences would lead to the most accurate results. This would be the conclusion based on formal identities between the derivative operator $\mathbf{D}^{r}$, defined by

$$
\mathbf{D}^{r} I_{i}=\left(\frac{\mathbf{D}^{r} I(x)}{d x^{r}}\right)_{x=(i-1) \Delta x}
$$

and the various shifting operators $\Delta^{r}, \underset{\sim}{\nabla^{r}}$, and $\boldsymbol{\delta}^{r}$.

From $\mathbf{E}=e^{\mathbf{D}}$ (in units employed in this work, based on arbitrary setting of $\Delta x=1$ ) we have, to the first approximation,

$$
\mathbf{D}^{r}=(\log \mathbf{E})^{r} \cong \boldsymbol{\Delta}^{r}\left(1-\frac{r}{2} \boldsymbol{\Delta}\right) \cong \boldsymbol{\nabla}^{r}(1+r / 2 \boldsymbol{\nabla}) \cong \boldsymbol{\delta}^{r}\left(1-\frac{\boldsymbol{\delta}^{2}}{2.3 !}\right)
$$

Equation (39) shows the magnitude of the error involved in replacing an $r$-order derivative by various 
$r$-order differences. It is the method, based on central differences, which leads to the standard integration formulas. For example, with $l=2$ and $p=2$, the coefficients in expansion (31) are the same as in Simpson's rule for numerical integration. With $l=p=6$, Weddle's rule is obtained. Unfortunately, the direct solution of the integral equation, based on the central-difference method, was shown to be numerically unstable. If, however, we wish to use the central difference method, we will have to employ other, indirect approaches to the algebraic solution of the integral equation. These approaches will be discussed now.

We start with

$$
\begin{aligned}
\sum_{j=i, i+1, \ldots}^{j=N} b_{i, j} I_{j} & \equiv \sum_{j=i, i+l, i+2 l, \ldots}^{j=N} I_{j} \int_{(j-1-l / 2) \Delta x}^{(j-1+l / 2) \Delta x} K[(i-1) \Delta x, x] d x \\
& +1 / 2\left[I_{j+1}-2 I_{j}+I_{j-1}\right] \int_{(j-1-l / 2) \Delta x}^{(j-1+l / 2) \Delta x}(x-j-1)^{2} K[(i-1) \Delta x, x] d x+\ldots=h_{i} .
\end{aligned}
$$

In matrix form, $\mathbf{B I}=\mathbf{h}$. Expand $\mathbf{B}=\mathbf{B}_{0}+\mathbf{B}_{2}+\mathbf{B}_{4}+\ldots$ The elements of the matrix $\mathbf{B}_{0}, b_{0}(i, j)$, are

$$
b_{0}(i, j)=\int_{(j-1-l / 2) \Delta x}^{(j-1+l / 2) \Delta x} K[(i-1) \Delta x, x] d x
$$

for $j=i, i+l, i+2 l, \ldots$ and $b_{0}(i, j)=0$ for all other values of $j . \mathbf{B}_{2}$ is the matrix of $b_{2}(i, j)$, and

$$
\begin{array}{r}
b_{2}(i, j)=-\int_{(j-l / 2-1) \Delta x}^{(j+l / 2-1) \Delta x}[x-(j-1) \Delta x]^{2} K[(i-l) \Delta x, x] d x \text { for } j=i, i+l, i+2 l, \\
b_{2}(i, j)=+\int_{(j-1-l / 2) \Delta x}^{(j-1+l / 2) \Delta x}(x-j \Delta x)^{2} K[(i-1) \Delta x, x] d x
\end{array}
$$

for $j=i+1, i+l+1 \ldots$,

$$
b_{2}(i, j)=1 / 2 \int_{(j-1-l / 2) \Delta x}^{(j-1+l / 2) \Delta x}[x-(j-1) \Delta x]^{2} K[(i-1) \Delta x, x] d x
$$

for $j=i-1$ and $b_{2}(i, j)=0$ for all other values of $j$. Similarly expressions for $b_{4}(i, j)$, etc., can be formulated. Let the zeroth-order solution be

$$
\mathbf{B}_{0} \mathbf{I}^{(0)}=\mathbf{h} .
$$

Application of perturbation theory leads to the following:

$$
\begin{gathered}
\mathbf{B}=\mathbf{B}_{0}+\lambda \mathbf{B}_{2}+\lambda^{2} \mathbf{B}_{4}+\ldots \\
\mathbf{I}=\mathbf{I}^{(1)}+\lambda \mathbf{I}^{(1)}+\lambda^{2} \mathbf{I}^{(2)}+\ldots
\end{gathered}
$$

Equating equal powers of $\lambda$ in equation $\mathbf{B I}=\mathbf{h}$ leads to the following expressions for $\mathbf{I}^{(1)}$ and $\mathbf{I}^{(2)}$ :

$$
I^{(1)}=-\mathbf{B}_{0}^{-1} \mathbf{B}_{2} \mathbf{I}^{(0)}
$$

and

$$
\begin{aligned}
\mathbf{I}_{1} & =\mathbf{I}^{(0)}+\mathbf{I}^{(1)} . \\
\mathbf{I}^{(2)} & =-\mathbf{B}_{0}^{-1}\left[\mathbf{B}_{4} \mathbf{I}^{(0)}+\mathbf{B}_{2} \mathbf{I}^{(1)}\right] .
\end{aligned}
$$


Since the zeroth order solution by definition, satisfies the conditions for numerical stability, the elements of the matrix $\mathbf{B}_{0}^{-1}$ are bounded. Specifically, for $K[(i-1) \Delta x, x]=x$ and $l=2, \mathbf{B}_{0}^{-1}$ is is of the form

$$
\mathbf{B}_{0}^{-1}=\left(\begin{array}{rrrrr}
1 / 2 & 0 & -1 / 2 & 0 & 0 . \\
0 & 1 / 4 & 0 & -1 / 4 & 0 . \\
0 & 0 & 1 / 6 & 0 & -1 / 6 \ldots \\
& & . & . & \ldots . \\
. & . & . & . & \ldots . \\
. & . & . & . & .
\end{array}\right)
$$

the matrix $\mathbf{B}_{2}$ is

$$
\mathbf{B}_{2}=1 / 6\left(\begin{array}{rrrrrr}
-2 & 4 & -6 & 8 & -10 & . . \\
2 & -4 & 6 & -8 & 10 & . \\
0 & 3 & -6 & 8 & -10 & . \\
0 & 0 & 4 & -8 & 10 & . \\
0 & 0 & 0 & 5 & -10 & . \\
. & . & . & . & . & . \\
. & . & . & . & . & . \\
. & . & . & . & . & .
\end{array}\right)
$$

We obtain for the correction term, $I_{i}^{(1)}$, the following relation:

$$
I_{i}^{(1)}=-1 / 12\left[I_{i-1}^{(0)}-2 I_{i}^{(0)}+I_{i+1}^{(0)}\right] .
$$

Thus, the correction to the zeroth order approximation is evaluated by calculating the second order differences, $\delta^{2} I_{i}^{(0)}$ of the zeroth-order solution, and subtracting $1 / 12 \delta^{2} I_{i}^{(0)}$ from $I_{i}^{(0)}$.

The above scheme does not exhaust all possibilities in applying the perturbation method. One can use the zeroth-order solution based on a trapezoid rule. The $\mathbf{B}_{2}$ matrix will then be constructed differently than the one given in the above example. However, a typical row of the inverse matrix $\mathbf{B}^{-1}$ (again, calculated for $K[(i-1) \Delta x, x] \cong x$ and for $i \gg 1$ ) will be

$$
\frac{2}{i}[1,-2,2,-2, \ldots]
$$

resulting in even-odd oscillations in the computed values of $I_{i}$. This is also known as weak numerical instability. This weak instability can be greatly reduced, if certain averaging procedures are adopted. For example, one can replace, $I_{i}^{(1)}=\sum_{j-i} \beta^{*}(i, j) I_{j}$, where $\beta^{*}(i, j)$ are the elements of matrix $\mathbf{B}_{0}^{-1} \mathbf{B}_{2}$, by $\overline{\mathbf{I}}^{(1)}=\mathbf{D I}$, where $\delta(i, j)$, the $(i, j)$ element of the matrix $\mathbf{D}$ is given by $\delta(i, j)=$

$$
\frac{1}{2}\left[\beta^{*}(i, j)+\beta^{*}(i+1, j)\right] .
$$

In summary, two methods are at our disposition:

(1) A multi-step method with forward differences, referred to the point being computed and extended to the points already computed. This method requires precalculated "starting" values. This number of starting points is usually equal to the multiplicity of the method. These starting values are most conveniently computed using a single-step method.

(2) A single-step method used as zeroth-order approximation. Higher-order approximations are then computed by a perturbation method. The advantage of this procedure is in employment of 
the more accurate central differences replacing the various derivatives. This method, if employed in combination with certain averaging procedures, tends to remove some of the weak instabilities (in form of even-odd oscillations) from the zeroth-order solution.

\section{Some Computational Details}

The two approaches outlined above, one based on a multi-step method and the other based on a single-step method serving as a zeroth order approximation in perturbation method, were employed in writing a general computer program (in Fortran) for the problem of numerical solution of eq (1). The first approach employs eq (34) as a starting equation for the actual computations. This equation is employed to the approximation that only terms up to $\Delta^{2}$ are retained in the expansion of the bracketed terms of eq (34). In the second approach the corrections to the zeroth order solution (which is given by eq (41)), were computed, by using eq (46) and also by performing averaging indicated by eq (47).

In actual computations, four different forms for the slit weighting function were used:

1. Constant weighting function (infinite slit)

2. Gaussian

3. Trapezoid, and

4. Data weighting function.

For the first three functions, parameters which describe them have to be supplied in the form of an experimentally determined set of values taken at a given constant interval. The kernel of the integral equation (given by eq (2a)) for these first three functions, is evaluated analytically. Moreover, all the required moments of the kernel (that is, integrals of the form $\int x^{t} K[(i-1) \Delta x, x] d x$, are also evaluated analytically. For the last assumed form for the weighting function, however, the computations of the kernel of the integral equation and its moments, have to be performed numerically. The time required to perform the entire "unsmearing" of the data is considerably longer for the case of the data-weighting function, than for either one of the other three functional forms of this function.

To our knowledge, the slit-weighting functions (1-3) are the only functions, which describe the actually measured weighting functions reasonably well, and which are also analytically tractable.

The results of the computations were subsequently tested by an inversion of the calculation procedures as follows: Using the calculated values of $\mathbf{I}$, the values of $\tilde{\tilde{\mathbf{I}}}$ were recalculated and compared with their original values. The accuracy of the numerical procedure was tested both with various trial functions for which analytical methods for solution of the original integral equations are available, and with experimental data taken from various measurements. Both approaches to the solution of the starting integral equation were tried with satisfactory results.

The author thanks R. J. Rubin for helpful discussions and suggestions in preparation of this publication. 


\section{References}

[1] Mazur, J., and Wims, A. M., J. Res. Nat. Bur. Stand. (U.S.), 70A (Phys. and Chem.) No. 6, 467-471 (Nov.-Dec. 1966).

[2] Shull, C. G., and Roess, L. C., J. Appl. Phys. 1 8, 295 (1947).

[3] Kratky, O., and Skala, Z., Zeit. Elektrochem. 62, 73 (1958).

[4] Kratky, O., Porod, G., and Skala, Z., Acta Physica, XIII, 96 (1960).

[5] Hendricks, R., and Schmidt, P., Acta Phys. Austriaca 26, 97 (1967).

[6] Fox, L., An Introduction to Linear Matrix Algebra (Oxford University Press, 1965), p. 32.
[7] For a general discussion of multi-step method, see Henrici, P., Discrete Variable Methods in Ordinary Differential Equations (John Wiley \& Sons, Inc., New York, 1962).

[8] Reference [7], p. 218, Theorem 5.5.

[9] Reference [7], Chapter 5.7.

[10] Hildebrand, F., Finite Difference Equations and Simulations, Chapter 2 (Prentice-Hall, Inc., Englewood Cliffs, N.J., 1968).

[11] Reference [7], p. 199.

(Paper 75B3\&4-357) 